\title{
MEASUREMENT OF RELIABILITY PARAMETERS FOR A POWER PLANT BY USING ALGEBRA OF LOGICS
}

\author{
Reena Garg ${ }^{1}$ \\ ${ }^{I}$ Assistant Professor Mathematics, YMCA University of Science \& Technology, Faridabad - 121006
}

\begin{abstract}
In this paper, the author has considered a power plant to evaluate its reliability parameters by using algebra of logics. The system configuration has been shown in fig-1. In this power plant, there are two identical generators $G_{1}$ and $G_{2}$ in standby redundancy. Only one generator is sufficient to fulfill the power requirements of whole system. The author has used four change over devices $\mathrm{CO}_{1}$ through $\mathrm{CO}_{4}$ to feed power to four machine $\mathrm{M}_{1}, M_{2}, M_{3}$ and $M_{4}$.It is assumed that the cables used, to connect all equipments, are hundred percent reliable and we need to operate two machines $M_{1}, M_{2}$, or $M_{3}$ and $M_{4}$ simultaneously. These machines can be operated by switching devices $S D_{1}$ through $S D_{4}$.
\end{abstract}

Keywords: power plant, identical generators, standby redundancy, changes over devices.

\section{INTRODUCTION}

In the beginning, the whole power plant is operable and there is no repair facility to repair a failed unit/component of power plant. The author has obtained the reliability of the system as a whole and reliability function for the system in two cases, i.e., when failure rates follow weibull or exponential time distribution. An important reliability parameter, mean time to failure (M.T.T.F.), has also been evaluated in both the cases. In the end of paper, the author has been given a numerical computation and its graphical illustration to highlight the important results for their practical importance. The results obtained in this study, can be applied for the power plant of similar configuration.

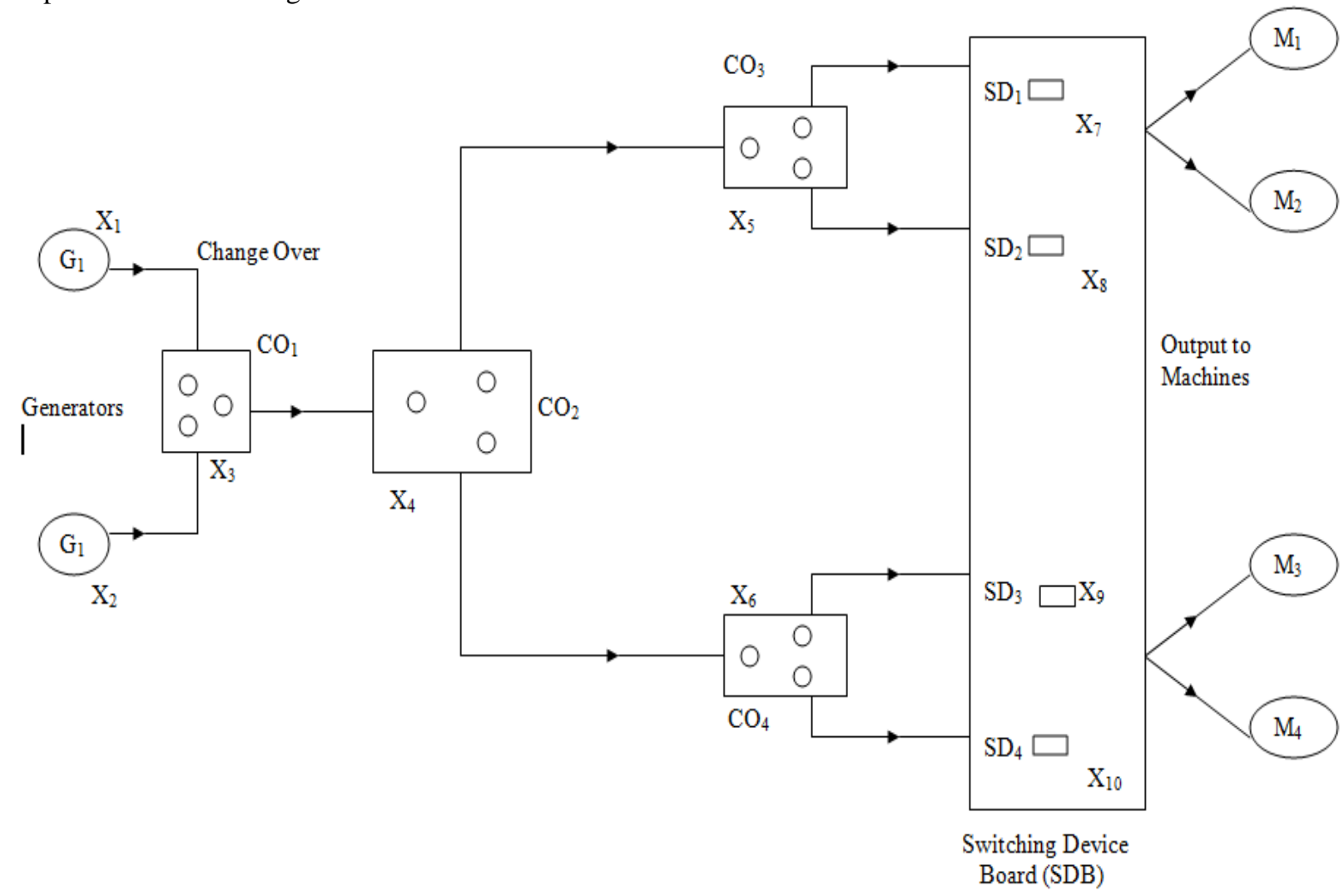

Fig-1: System Configuration 


\section{ASSUMPTIONS}

The following assumptions have been associated with this model:

(1) Initially, all the components are good.

(2) There is no repair facility available.

(3) Failure times of all components of the power plant are arbitrary.

(4) Failures are statistically-independent.

(5) Reliability of each component of power plant is known in advance.

(6) Transition of power from one component to other is all reliable.

(7) Every component of the system remains either in good or in bad state.

(8) Only one generator is sufficient to fullfil the power requirement of whole power plant.

(9) Mostly, we need two machines $M_{1}$ and $M_{2}$ or $M_{3}$ and $\mathrm{M}_{4}$ to operate simultaneously.

\section{NOTATIONS}

The following notations have been used throughout this paper:

$x_{1}, x_{2}$

States of generators $G_{1}, G_{2}$ respectively;

$x_{3}, x_{4}, x_{5}, x_{6}:$ States of change over devices $\mathrm{CO}_{1}$ through $\mathrm{CO}_{4}$;

$x_{7}, x_{8}, x_{9}, x_{10}:$ States of switching devices $S D_{1}$ through $\mathrm{SD}_{4}$;

$x_{i}^{\prime} \quad: \quad$ Negation of state $x_{i}, \forall i$;

$x_{i} \quad: \quad=1$, in good state; $=0$ in bad state;

$\wedge / \vee \quad$ : Conjunction/Disjunction;

$R_{i} \quad:$ Reliability of the component corresponding to state $x_{i}$;

$S_{i} \quad:=1-R_{i}, \forall i$

$R_{S} \quad: \quad$ Reliability of the power plant as a $R_{S E}(t) / R_{S W}(t) \quad: \begin{aligned} & \text { whole; } \\ & \text { Reliability functions when failures } \\ & \text { follow Weibull/exponential time }\end{aligned}$ distribution.

\section{FORMULATION OF MATHEMATICAL MODEL}

By making use of Boolean function technique, the conditions of capability of the successful operation of the power plant in terms of logical matrix are expressed as:

$$
F\left(x_{1}, x_{2}--x_{10}\right)=\left[\begin{array}{ccccc}
x_{1} & x_{3} & x_{4} & x_{5} & x_{7} \\
x_{1} & x_{3} & x_{4} & x_{5} & x_{8} \\
x_{1} & x_{3} & x_{4} & x_{6} & x_{9} \\
x_{1} & x_{3} & x_{4} & x_{6} & x_{10} \\
x_{2} & x_{3} & x_{4} & x_{5} & x_{7} \\
x_{2} & x_{3} & x_{4} & x_{5} & x_{8} \\
x_{2} & x_{3} & x_{4} & x_{6} & x_{9} \\
x_{2} & x_{3} & x_{4} & x_{6} & x_{10}
\end{array}\right]
$$

\section{SOLUTION OF THE MODEL}

Using algebra of logics, we may write equation (1) as:

$$
F\left(x_{1}, x_{2}--x_{10}\right)=\left[\begin{array}{ll}
x_{3} & x_{4}
\end{array}\right] \wedge f\left(x_{1}, x_{2}, x_{5}--, x_{10}\right),
$$

Where

$$
f\left(x_{1}, x_{2}, x_{5}--, x_{10}\right)=\left[\begin{array}{ccc}
x_{1} & x_{5} & x_{7} \\
x_{1} & x_{5} & x_{8} \\
x_{1} & x_{6} & x_{9} \\
x_{1} & x_{6} & x_{10} \\
x_{2} & x_{5} & x_{7} \\
x_{2} & x_{5} & x_{8} \\
x_{2} & x_{6} & x_{9} \\
x_{2} & x_{6} & x_{10}
\end{array}\right]
$$

Substituting

$$
\begin{aligned}
& B_{1}=\left[\begin{array}{lll}
x_{1} & x_{5} & x_{7}
\end{array}\right] \\
& B_{2}=\left[\begin{array}{lll}
x_{1} & x_{5} & x_{8}
\end{array}\right] \\
& B_{3}=\left[\begin{array}{lll}
x_{1} & x_{6} & x_{9}
\end{array}\right] \\
& B_{4}=\left[\begin{array}{lll}
x_{1} & x_{6} & x_{10}
\end{array}\right] \\
& B_{5}=\left[\begin{array}{lll}
x_{2} & x_{5} & x_{7}
\end{array}\right] \\
& B_{6}=\left[\begin{array}{lll}
x_{2} & x_{5} & x_{8}
\end{array}\right] \\
& B_{7}=\left[\begin{array}{lll}
x_{2} & x_{6} & x_{9}
\end{array}\right] \\
& B_{8}=\left[\begin{array}{lll}
x_{2} & x_{6} & x_{10}
\end{array}\right]
\end{aligned}
$$


in equation (3), we have

$$
f\left(x_{1}, x_{2}, x_{5}-, x_{10}\right)=\left[\begin{array}{c}
B_{1} \\
B_{2} \\
B_{3} \\
B_{4} \\
B_{5} \\
B_{6} \\
B_{7} \\
B_{8}
\end{array}\right]
$$

Using orthogonalisation algorithm, equation (12) may be written as

$$
f\left(x_{1}, x_{2}, x_{5}--, x_{10}\right)=\left[\begin{array}{lllllllllll}
B_{1} & & & & & & & \\
B_{1}^{\prime} & B_{2} & & & & & & \\
B_{1}^{\prime} & B_{2}^{\prime} & B_{3} & & & & & \\
B_{1}^{\prime} & B_{2}^{\prime} & B_{3}^{\prime} & B_{4} & & & & \\
B_{1}^{\prime} & B_{2}^{\prime} & B_{3}^{\prime} & B_{4}^{\prime} & B_{5} & & & \\
B_{1}^{\prime} & B_{2}^{\prime} & B_{3}^{\prime} & B_{4}^{\prime} & B_{5}^{\prime} & B_{6} & & \\
B_{1}^{\prime} & B_{2}^{\prime} & B_{3}^{\prime} & B_{4}^{\prime} & B_{5}^{\prime} & B_{6}^{\prime} & B_{7} & \\
B_{1}^{\prime} & B_{2}^{\prime} & B_{3}^{\prime} & B_{4}^{\prime} & B_{5}^{\prime} & B_{6}^{\prime} & B_{7}^{\prime} & B_{8}
\end{array}\right]
$$

Now we have

$$
B_{1}^{\prime}=\left[\begin{array}{ccc}
x_{1}^{\prime} & & \\
x_{1} & x_{5}^{\prime} & \\
x_{1} & x_{5} & x_{7}^{\prime}
\end{array}\right]
$$

$\therefore B_{1}^{\prime} B_{2}=\left[\begin{array}{ccc}x_{1}^{\prime} & & \\ x_{1} & x_{5}^{\prime} & \\ x_{1} & x_{5} & x_{7}^{\prime}\end{array}\right] \wedge\left[\begin{array}{lll}x_{1} & x_{5} & x_{8}\end{array}\right]$

Therefore, equation (13) becomes, subjected to equations (14) through (20):

$$
f\left(x_{1}, x_{2}, x_{5}--, x_{10}\right)=\left[\begin{array}{ccccccccc}
x_{1} & x_{5} & x_{7} & & & & & \\
x_{1} & x_{5} & x_{7}^{\prime} & x_{8} & & & & \\
x_{1} & x_{5}^{\prime} & x_{6} & x_{9} & & & & \\
x_{1} & x_{5} & x_{6} & x_{7}^{\prime} & x_{8} & x_{9} & & \\
x_{1} & x_{5}^{\prime} & x_{6} & x_{9}^{\prime} & x_{10} & & & \\
x_{1} & x_{5} & x_{6} & x_{7}^{\prime} & x_{8} & x_{9}^{\prime} & x_{10} & \\
x_{1}^{\prime} & x_{2} & x_{5} & x_{7} & & & & \\
x_{1}^{\prime} & x_{2} & x_{5} & x_{7}^{\prime} & x_{8} & & & \\
x_{1} & x_{2} & x_{5} & x_{6}^{\prime} & x_{7}^{\prime} & x_{8} & & \\
x_{1} & x_{2} & x_{5} & x_{6} & x_{7}^{\prime} & x_{8} & x_{9}^{\prime} & x_{10} \\
x_{1}^{\prime} & x_{2} & x_{5}^{\prime} & x_{6} & x_{9} & & & \\
x_{1}^{\prime} & x_{2} & x_{5} & x_{6} & x_{7}^{\prime} & x_{8}^{\prime} & x_{9} & \\
x_{1}^{\prime} & x_{2} & x_{5}^{\prime} & x_{6} & x_{9}^{\prime} & x_{10} & & \\
x_{1}^{\prime} & x_{2} & x_{5} & x_{6} & x_{7}^{\prime} & x_{8}^{\prime} & x_{9}^{\prime} & x_{10}
\end{array}\right]
$$


Using equation (21), equation (2) gives

$$
F\left(x_{1}, x_{2}--, x_{10}\right)=\left[\begin{array}{lllllllllll}
x_{1} & x_{3} & x_{4} & x_{5} & x_{7} & & & & \\
x_{1} & x_{3} & x_{4} & x_{5} & x_{7}^{\prime} & x_{8} & & & \\
x_{1} & x_{3} & x_{4} & x_{5}^{\prime} & x_{6} & x_{9} & & & \\
x_{1} & x_{3} & x_{4} & x_{5} & x_{6} & x_{7}^{\prime} & x_{8} & x_{9} & & \\
x_{1} & x_{3} & x_{4} & x_{5}^{\prime} & x_{6} & x_{9}^{\prime} & x_{10} & & & \\
x_{1} & x_{3} & x_{4} & x_{5} & x_{6} & x_{7}^{\prime} & x_{8} & x_{9}^{\prime} & x_{10} & \\
x_{1}^{\prime} & x_{2} & x_{3} & x_{4} & x_{5} & x_{7} & & & & \\
x_{1}^{\prime} & x_{2} & x_{3} & x_{4} & x_{5} & x_{7}^{\prime} & x_{8} & & & \\
x_{1} & x_{2} & x_{3} & x_{4} & x_{5} & x_{6}^{\prime} & x_{7}^{\prime} & x_{8} & & \\
x_{1} & x_{2} & x_{3} & x_{4} & x_{5} & x_{6} & x_{7}^{\prime} & x_{8} & x_{9}^{\prime} & x_{10} \\
x_{1}^{\prime} & x_{2} & x_{3} & x_{4} & x_{5}^{\prime} & x_{6} & x_{9} & & & \\
x_{1}^{\prime} & x_{2} & x_{3} & x_{4} & x_{5} & x_{6} & x_{7}^{\prime} & x_{8}^{\prime} & x_{9} & \\
x_{1}^{\prime} & x_{2} & x_{3} & x_{4} & x_{5}^{\prime} & x_{6} & x_{9}^{\prime} & x_{10} & & \\
x_{1}^{\prime} & x_{2} & x_{3} & x_{4} & x_{5} & x_{6} & x_{7}^{\prime} & x_{8}^{\prime} & x_{9}^{\prime} & x_{10}
\end{array}\right]
$$

Since, R.H.S. of equation (23) is disjunction of pair-wise disjoint conjunctions, therefore, the reliability of considered power plant will be:

$$
\begin{aligned}
& R_{S}=P_{r}\left\{F\left(x_{1}, x_{2}--x_{10}\right)=1\right\} \\
& =R_{3} R_{4}\left[R_{1} R_{5} R_{7}+R_{1} R_{5} R_{8} S_{7}+R_{1} R_{6} R_{9} S_{5}+R_{1} R_{5} R_{6} R_{8} R_{9} S_{7}+S_{5} S_{9} R_{1} R_{6} R_{10}\right. \\
& +S_{7} S_{9} R_{1} R_{5} R_{6} R_{8} R_{10}+S_{1} R_{2} R_{5} R_{7}+S_{1} S_{7} R_{2} R_{5} R_{8}+S_{6} S_{7} R_{1} R_{2} R_{5} R_{8} \\
& +S_{1} S_{5} R_{2} R_{6} R_{9}+S_{7} S_{9} R_{1} R_{2} R_{5} R_{6} R_{8} R_{10}+S_{1} S_{7} S_{8} R_{2} R_{5} R_{6} R_{9} \\
& \left.+S_{1} S_{5} S_{9} R_{2} R_{6} R_{10}+S_{1} S_{7} S_{8} S_{9} R_{2} R_{5} R_{6} R_{10}\right]
\end{aligned}
$$

Where, $S_{i}=1-R_{i}, \forall i=1,2,---10$.

or, $\quad R_{S}=R_{3} R_{4}\left[R_{1} R_{5} R_{7}+R_{1} R_{5} R_{8}+R_{1} R_{6} R_{9}+R_{2} R_{5} R_{7}+R_{1} R_{6} R_{10}+R_{2} R_{5} R_{8}\right.$

$+R_{2} R_{6} R_{9}+R_{2} R_{6} R_{10}+R_{1} R_{5} R_{6} R_{8} R_{9}+R_{1} R_{5} R_{6} R_{9} R_{10}+R_{1} R_{5} R_{6} R_{8} R_{10}$

$+R_{1} R_{5} R_{6} R_{7} R_{8} R_{9} R_{10}+R_{1} R_{2} R_{5} R_{6} R_{7} R_{8}+R_{1} R_{2} R_{5} R_{6} R_{8} R_{10}$

$+R_{1} R_{2} R_{5} R_{6} R_{7} R_{8} R_{9} R_{10}+R_{1} R_{2} R_{5} R_{6} R_{7} R_{9}+R_{1} R_{2} R_{5} R_{6} R_{8} R_{9}+R_{2} R_{5} R_{6} R_{7} R_{8} R_{9}$

$+R_{1} R_{2} R_{6} R_{9} R_{10}+R_{1} R_{2} R_{5} R_{6} R_{7} R_{10}+R_{1} R_{2} R_{5} R_{6} R_{8} R_{10}+R_{2} R_{5} R_{6} R_{7} R_{8} R_{10}$

$+R_{2} R_{5} R_{6} R_{7} R_{9} R_{10}+R_{2} R_{5} R_{6} R_{8} R_{9} R_{10}+R_{1} R_{2} R_{5} R_{6} R_{7} R_{8} R_{9} R_{10}$

$-R_{1} R_{5} R_{7} R_{8}-R_{1} R_{5} R_{6} R_{9}-R_{1} R_{5} R_{6} R_{7} R_{8} R_{9}-R_{1} R_{2} R_{5} R_{7}-R_{1} R_{5} R_{6} R_{10}$ 
$-R_{1} R_{6} R_{9} R_{10}-R_{1} R_{5} R_{6} R_{7} R_{8} R_{10}-R_{1} R_{5} R_{6} R_{8} R_{9} R_{10}-R_{2} R_{5} R_{7} R_{8}-R_{1} R_{2} R_{5} R_{6} R_{8}$

$-R_{1} R_{2} R_{5} R_{6} R_{7} R_{8} R_{10}-R_{1} R_{2} R_{5} R_{6} R_{8} R_{9} R_{10}-R_{1} R_{2} R_{6} R_{9}-R_{2} R_{5} R_{6} R_{7} R_{9}$

$-R_{2} R_{5} R_{6} R_{8} R_{9}-R_{1} R_{2} R_{5} R_{6} R_{7} R_{8} R_{9}-R_{1} R_{2} R_{6} R_{10}-R_{2} R_{6} R_{9} R_{10}-R_{2} R_{5} R_{6} R_{7} R_{10}$

$-R_{2} R_{5} R_{6} R_{8} R_{10}-R_{1} R_{2} R_{5} R_{6} R_{7} R_{8} R_{10}-R_{1} R_{2} R_{5} R_{6} R_{7} R_{9} R_{10}-R_{1} R_{2} R_{5} R_{6} R_{8} R_{9} R_{10}$

$\left.-R_{2} R_{5} R_{6} R_{7} R_{8} R_{9} R_{10}\right]$

\section{SOME PARTICULAR CASES}

6.1 CASE I: If the Reliability of each Component of Power Plant is R:

In this case, equation (24) yields:

$$
R_{S}=8 R^{5}-9 R^{6}-R^{7}+7 R^{8}-6 R^{9}+2 R^{10}
$$

\subsection{CASE II: When Failure Rates follow Weibull}

\section{Distribution}

Let $\lambda_{i}$ be the failure rates corresponding to component states $x_{i}, \forall i=1,2---10$. Then, reliability function of power plant, at an instant ${ }^{\prime} \mathrm{t}$, is given by:

$$
R_{S W}(t)=\sum_{i=1}^{25} \exp \cdot\left\{-a_{i} t^{\alpha}\right\}-\sum_{j=1}^{24} \exp .\left\{-b_{j} t^{\alpha}\right\}
$$

$$
\text { where, } \alpha \text { is a positive parameter and }
$$

$$
\begin{aligned}
& a_{1}=c+\lambda_{1}+\lambda_{5}+\lambda_{7} \\
& a_{2}=c+\lambda_{1}+\lambda_{5}+\lambda_{8} \\
& a_{3}=c+\lambda_{1}+\lambda_{6}+\lambda_{9} \\
& a_{4}=c+\lambda_{1}+\lambda_{5}+\lambda_{6}+\lambda_{8}+\lambda_{9} \\
& a_{5}=c+\lambda_{2}+\lambda_{5}+\lambda_{7} \\
& a_{6}=c+\lambda_{1}+\lambda_{6}+\lambda_{10} \\
& a_{7}=c+\lambda_{1}+\lambda_{5}+\lambda_{6}+\lambda_{9}+\lambda_{10} \\
& a_{8}=c+\lambda_{1}+\lambda_{5}+\lambda_{6}+\lambda_{8}+\lambda_{10}
\end{aligned}
$$

$$
\begin{aligned}
& a_{9}=c+\lambda_{1}+\lambda_{5}+\lambda_{6}+\lambda_{7}+\lambda_{8}+\lambda_{9}+\lambda_{10} \\
& a_{10}=c+\lambda_{2}+\lambda_{5}+\lambda_{8} \\
& a_{11}=c+\lambda_{1}+\lambda_{2}+\lambda_{5}+\lambda_{6}+\lambda_{7}+\lambda_{8} \\
& a_{12}=c+\lambda_{1}+\lambda_{2}+\lambda_{5}+\lambda_{6}+\lambda_{8}+\lambda_{10}
\end{aligned}
$$$$
a_{13}=c+\lambda_{1}+\lambda_{2}+\lambda_{5}+\lambda_{6}+\lambda_{7}+\lambda_{8}+\lambda_{9}+\lambda_{10}
$$$$
a_{14}=c+\lambda_{2}+\lambda_{6}+\lambda_{9}
$$$$
a_{15}=c+\lambda_{1}+\lambda_{2}+\lambda_{5}+\lambda_{6}+\lambda_{7}+\lambda_{9}
$$$$
a_{16}=c+\lambda_{1}+\lambda_{2}+\lambda_{5}+\lambda_{6}+\lambda_{8}+\lambda_{9}
$$$$
a_{17}=c+\lambda_{2}+\lambda_{5}+\lambda_{6}+\lambda_{7}+\lambda_{8}+\lambda_{9}
$$$$
a_{18}=c+\lambda_{2}+\lambda_{6}+\lambda_{10}
$$$$
a_{19}=c+\lambda_{1}+\lambda_{2}+\lambda_{6}+\lambda_{9}+\lambda_{10}
$$$$
a_{20}=c+\lambda_{1}+\lambda_{2}+\lambda_{5}+\lambda_{6}+\lambda_{7}+\lambda_{10}
$$$$
a_{21}=c+\lambda_{1}+\lambda_{2}+\lambda_{5}+\lambda_{6}+\lambda_{8}+\lambda_{10}
$$$$
a_{22}=c+\lambda_{2}+\lambda_{5}+\lambda_{6}+\lambda_{7}+\lambda_{8}+\lambda_{10}
$$$$
a_{23}=c+\lambda_{2}+\lambda_{5}+\lambda_{6}+\lambda_{7}+\lambda_{9}+\lambda_{10}
$$$$
a_{24}=c+\lambda_{2}+\lambda_{5}+\lambda_{6}+\lambda_{8}+\lambda_{9}+\lambda_{10}
$$$$
a_{25}=c+\lambda_{1}+\lambda_{2}+\lambda_{5}+\lambda_{6}+\lambda_{7}+\lambda_{8}+\lambda_{9}+\lambda_{10}
$$

Also,

$b_{1}=c+\lambda_{1}+\lambda_{5}+\lambda_{7}+\lambda_{8}$ 
$b_{2}=c+\lambda_{1}+\lambda_{5}+\lambda_{6}+\lambda_{9}$

$b_{3}=c+\lambda_{1}+\lambda_{5}+\lambda_{6}+\lambda_{7}+\lambda_{8}+\lambda_{9}$

$b_{4}=c+\lambda_{1}+\lambda_{2}+\lambda_{5}+\lambda_{7}$

$b_{5}=c+\lambda_{1}+\lambda_{5}+\lambda_{6}+\lambda_{10}$

$b_{6}=c+\lambda_{1}+\lambda_{6}+\lambda_{9}+\lambda_{10}$

$b_{7}=c+\lambda_{1}+\lambda_{5}+\lambda_{6}+\lambda_{7}+\lambda_{8}+\lambda_{10}$

$b_{8}=c+\lambda_{1}+\lambda_{5}+\lambda_{6}+\lambda_{8}+\lambda_{9}+\lambda_{10}$

$b_{9}=c+\lambda_{2}+\lambda_{5}+\lambda_{7}+\lambda_{8}$

$b_{10}=c+\lambda_{1}+\lambda_{2}+\lambda_{5}+\lambda_{6}+\lambda_{8}$

$b_{11}=c+\lambda_{1}+\lambda_{2}+\lambda_{5}+\lambda_{6}+\lambda_{7}+\lambda_{8}+\lambda_{10}$

$b_{12}=c+\lambda_{1}+\lambda_{2}+\lambda_{5}+\lambda_{6}+\lambda_{8}+\lambda_{9}+\lambda_{10}$

$b_{13}=c+\lambda_{1}+\lambda_{2}+\lambda_{6}+\lambda_{9}$

$b_{14}=c+\lambda_{2}+\lambda_{5}+\lambda_{6}+\lambda_{7}+\lambda_{9}$

$b_{15}=c+\lambda_{2}+\lambda_{5}+\lambda_{6}+\lambda_{8}+\lambda_{9}$

$b_{16}=c+\lambda_{1}+\lambda_{2}+\lambda_{5}+\lambda_{6}+\lambda_{7}+\lambda_{8}+\lambda_{9}$

$b_{17}=c+\lambda_{1}+\lambda_{2}+\lambda_{6}+\lambda_{10}$

$b_{18}=c+\lambda_{2}+\lambda_{6}+\lambda_{9}+\lambda_{10}$

$b_{19}=c+\lambda_{2}+\lambda_{5}+\lambda_{6}+\lambda_{7}+\lambda_{10}$

$b_{20}=c+\lambda_{2}+\lambda_{5}+\lambda_{6}+\lambda_{8}+\lambda_{10}$

$b_{21}=c+\lambda_{1}+\lambda_{2}+\lambda_{5}+\lambda_{6}+\lambda_{7}+\lambda_{8}+\lambda_{10}$

$b_{22}=c+\lambda_{1}+\lambda_{2}+\lambda_{5}+\lambda_{6}+\lambda_{7}+\lambda_{9}+\lambda_{10}$

$b_{23}=c+\lambda_{1}+\lambda_{2}+\lambda_{5}+\lambda_{6}+\lambda_{8}+\lambda_{9}+\lambda_{10}$

$b_{24}=c+\lambda_{2}+\lambda_{5}+\lambda_{6}+\lambda_{7}+\lambda_{8}+\lambda_{9}+\lambda_{10}$

and $c=\lambda_{3}+\lambda_{4}$
Again, the M.T.T.F. of the power plant, in this case, is given by:

$$
\begin{aligned}
& M \cdot T \cdot T \cdot F .(W)=\int_{0}^{\infty} R_{S W}(t) d t \\
& =\frac{1}{\alpha} \Gamma\left(\frac{1}{\alpha}\right)\left[\sum_{i=1}^{25} \frac{1}{\left(a_{i}\right)^{1 / \alpha}}-\sum_{j=1}^{24} \frac{1}{\left(b_{j}\right)^{1 / \alpha}}\right]
\end{aligned}
$$

\subsection{CASE III: When Failures follow Exponential}

\section{Distribution}

Exponential distribution is nothing but a particular case of weibull distribution for $\alpha=1$ and is very useful for practical problem purpose. Therefore, the reliability function for considered power plant at an instant $t$, in this case, is given by

$$
R_{S E}(t)=\sum_{i=1}^{25} \exp .\left\{-a_{i} t\right\}-\sum_{j=1}^{24} \exp .\left\{-b_{j} t\right\}
$$

and expression for M.T.T.F., in this case, is given by

$$
\begin{aligned}
& \text { M.T.T.F.(E) }=\int_{0}^{\infty} R_{S E}(t) d t \\
& =\sum_{i=1}^{25}\left(\frac{1}{a_{i}}\right)-\sum_{j=1}^{24}\left(\frac{1}{b_{j}}\right)
\end{aligned}
$$

\section{NUMERICAL COMPUTATION}

For a numerical computation, let us consider the values:

(a) $\lambda_{i}(i=1,2--10)=\lambda=0.002, \alpha=2$ and $t=0,1,2--$ in equation (26);

(b) $\lambda_{i}(i=1,2--10)=\lambda=0.002, \quad t=0,1,2--$ in equation (28).

By using (a) and (b), we compute the table-1 and the corresponding graph has been shown in fig-2.

(c)

$$
\alpha=2
$$

and $\lambda_{i}(i=1,2--10)=\lambda=0,0.04,0.09--, 1$ in equation (27);

(d) $\quad \lambda_{i}(i=1,2--10)=\lambda=0,0.04,0.09--, 1$ in equation (29).

By using (c) and (d), we compute the table- 2 and the corresponding graph has been shown in fig- 3 . 
Table-1

\begin{tabular}{|l|l|l|}
\hline $\mathbf{t}$ & $\mathbf{R}_{\text {SW }}(\mathbf{t})$ & $\mathbf{R}_{\text {SE }}(\mathbf{t})$ \\
\hline 0 & 1 & 1 \\
\hline 1 & 0.9979 & 0.9979 \\
\hline 2 & 0.9917 & 0.9959 \\
\hline 3 & 0.9803 & 0.9938 \\
\hline 4 & 0.9628 & 0.9917 \\
\hline 5 & 0.9379 & 0.9895 \\
\hline 6 & 0.9044 & 0.9872 \\
\hline 7 & 0.8614 & 0.9849 \\
\hline 8 & 0.8088 & 0.9826 \\
\hline 9 & 0.7471 & 0.9803 \\
\hline 10 & 0.6778 & 0.9779 \\
\hline
\end{tabular}

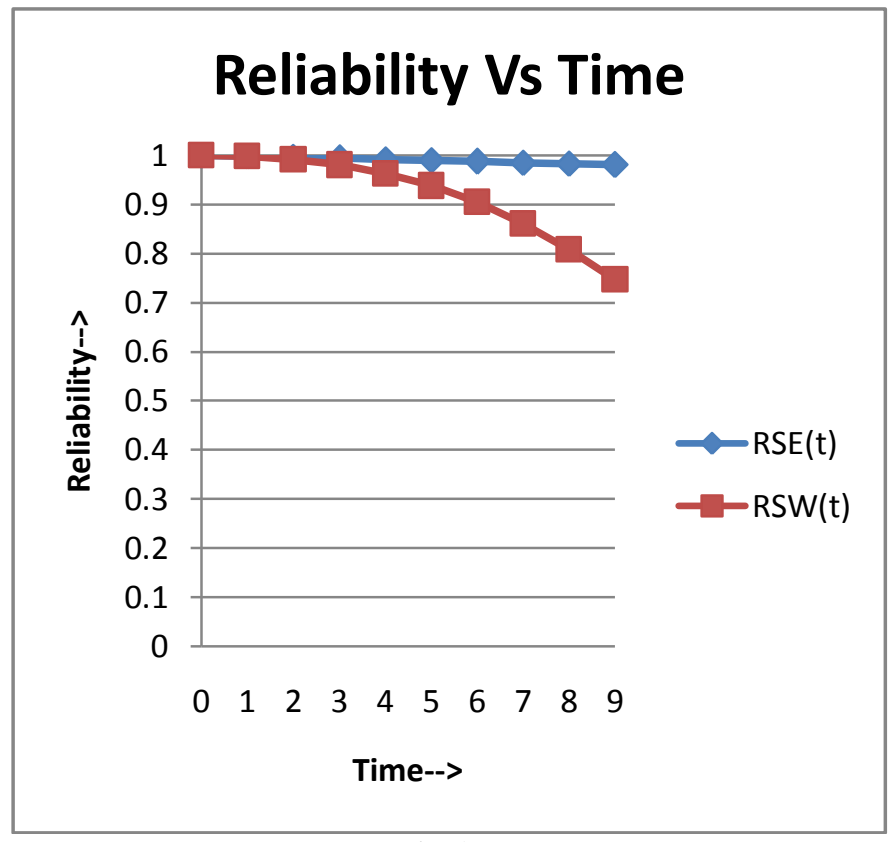

Fig-2

Table-2

\begin{tabular}{|l|l|l|}
\hline $\boldsymbol{\lambda}$ & M.T.T.F.(W) & M.T.T.F.(E) \\
\hline 0 & $\infty$ & $\infty$ \\
\hline 0.04 & 1.6198 & 9.1369 \\
\hline 0.09 & 1.0799 & 4.0608 \\
\hline 0.16 & 0.8099 & 2.2842 \\
\hline 0.25 & 0.6479 & 1.4619 \\
\hline 0.36 & 0.5399 & 1.0152 \\
\hline 0.49 & 0.4628 & 0.7459 \\
\hline 0.64 & 0.4049 & 0.5711 \\
\hline 0.81 & 0.3599 & 0.4512 \\
\hline 1.0 & 0.3239 & 0.3655 \\
\hline
\end{tabular}

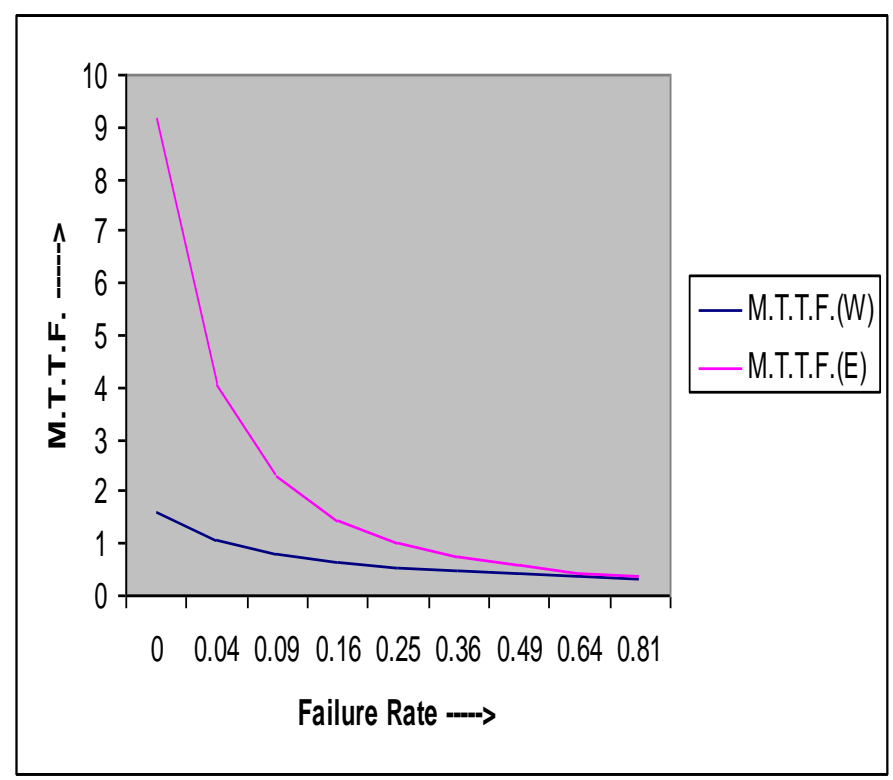

Fig-3

\section{RESULTS AND DISCUSSION}

In this study, the author has been obtained various performance measures of considered power plant, by using algebra of logics. The reliability and M.T.T.F. of power plant have been obtained in two different cases, i.e., when failures follow Weibull and exponential time distributions. The final results have been mentioned in the equations (25) through (29).

A critical examination of fig-2 and 3 shows that performance of considered system decreases with time. In case of Weibull time distribution, performance decreases catastrophically in the beginning but thereafter it decreases in a constant manner. We conclude in last that exponential time distribution gives better results as compared with Weibull time distribution.

\section{REFERENCES}

[1] Calabro, S.R.: "Reliability Principles and Practices", McGraw Hill Book Company, Inc. New York, 1962.

[2] Cinlar, E: "Introduction to Stochastic Processes", Prentice Hall Inc. Englewood cliffs, New Jersey, 1975.

[3] Culp, W. Archie: "Principles of Energy Conversion", McGraw Hill Book Company, New York, 2000.

[4] Dhillon, B.S.: "Quality Control, Reliability and Engineering Design”, Marcel Dekker, 1985.

[5] Dhillon, B.S.: "Design Reliability", CRC Press, 1999.

[6] Dhillon, B.S.: "Reliability, Quality and Safety Engineers", Taylor Francis, 2004.

[7] Degarmo, E.; Black, J.T.; Ronald, A.: "Materials and Processes in Manufacturing", Prentice Hall of India, 1997.

[8] Nagraja, H.N.; Kannan, N.; Krishnan, N.B.: "Reliability", Springer Publication, 2004. 
[9] Nikolaidis , E.; Ghiocel , D.M. ; Singhal , S. : "Engineering Design Reliability Handbook", CRC Press, 2004.

[10] N.Ravichandran ; "Stochastic Methods in Reliability Theory", Wiley Eastern Ltd; New Delhi, 1990.

[11] Nagrath, I.J.; Gopal, M.: "System Modeling and Analysis", Tata McGraw Hill Book Company, 1998.

[12] Osaki, S.: "Stochastic Models in Reliability and Maintenance", Springer Publication, 2002.

[13] Rao, Singiresu S.: "Engineering Optimization; Theory and Practice", New Age Int.(P) Limited, 1999.

[14] Rao, S.; Parulekar, B.B.: "Energy Technology", Khanna Publishers, New Delhi, 2002.

[15] Kuo, W; Prasad, V.R.; Tillman, F.A. and Hwang, C.L: "Fundamental and Applications of Reliability Optimization", Cambridge University Press, Cambridge, 2000.

[16] Chung, W. K.: "A k-out -of n: G Redundant System with the Presence of Chance with Multiple Critical Errors", Microelectronic Reliab., Vol. 33, pp 334-338, 1993.

[17] Chung, W. K.: " Reliability Analysis of a k-out -of n: G Redundant System in the Presence of Chance with Multiple Critical Errors", Microelectronic Reliab., Vol. 32, pp 331 - 334, 1993.

[18] Cluzeau, T.; Keller, J.; Schneeweiss, W.: “An Efficient Algorithm for Computing the Reliability of Consecutive-k-out-of-n:F Systems", IEEE TR. on Reliability, Vol.57 (1), PP 84-87, 2008.

[19] Dhillon, B. S.; Natesan, J.: "Stochastic Analysis of Outdoor Power System in Fluctuating Environment", Microelectronic Reliab. , Vol.23, pp $867-887,1983$.

[20] Dhillon, B. S.: "Reliability Analysis of NonMaintained Parallel System Subject to Hardware Failure and Human Error", Microelectronic Reliab., Vol. 25, pp 121 -122, 1985.

[21] Dhillon, B. S.; Vishvanath, H. C.: "Reliability Analysis of Non - identical Units Parallel System with Common Cause Failure", Microelectronic Reliab., Vol. 31, pp 429 - 441, 1991.

[22] Dhillon, B. S. and Yang N. J.: "Stochastic Analysis of Standby System with Common Cause Failure and Human Error", Microelectronic Reliab. , Vol. 32, pp 1699 - 1712, 1992.

[23] Dhillon, B. S. and Yang Nianfu: "Availability of a Man - Machine System with Critical and Noncritical Human Errors", Microelectronic Reliab. , Vol. 33 (10), pp 1511 - 1521, 1993. 